\title{
The Upgrade and Performance of the ATLAS Electron and Photon Triggers Towards Run 2
}

\author{
Joseph Reichert*, on behalf of the ATLAS Collaboration \\ University of Pennsylvania \\ E-mail: Joseph.Reichertecern.ch
}

\begin{abstract}
Electron and photon triggers are essential for signal selection in a wide variety of ATLAS physics analyses to study Standard Model processes and to search for new phenomena. Final states including leptons and photons had, for example, an essential role in the discovery and measurement of the Higgs particle. Dedicated triggers are also used for the collection of $J / \psi \rightarrow e e, W \rightarrow e v$, and QCD background samples for calibration, efficiency, and fake rate measurements. The ATLAS trigger system is divided in a hardware-based (Level 1) and a software-based high level trigger, both of which were upgraded during the long shutdown of the LHC in preparation for data taking in 2015. The increasing luminosity and more challenging pile-up conditions as well as the planned higher center-of-mass energy demanded the optimization of the trigger selections at each level to control the rates and keep efficiencies high. The evolution of the ATLAS electron and photon triggers and their performance will be presented, including initial results from the early days of the LHC Run 2 operation.
\end{abstract}

XXVII International Symposium on Lepton Photon Interactions at High Energies 17-22 August 2015

Ljubljana, Slovenia

${ }^{*}$ Speaker. 


\section{Introduction}

Electrons and photons were essential components of a wide variety of physics analyses on ATLAS [1] during Run 1 of the LHC. Among these were precision Standard Model measurements, searches for new physics, and the discovery and subsequent measurements of the Higgs Boson. Selection of events in real time (triggering) based on electron and photon signatures is more challenging in Run 2 of the LHC due to the higher center-of-mass energy and higher luminosity, which includes a larger number of overlapping inelastic proton-proton interactions (pile-up). These changes to the LHC beam conditions correspond to more difficult signal-to-background discrimination and higher trigger rates. To maintain the excellent performance achieved in Run 1, many upgrades and improvements have been made in preparation for Run 2 [2].

In the hardware-based Level 1 (L1) trigger, calorimeter information is used to select electromagnetic (EM) objects by identifying regions of interest and determining if a given cluster passes the $\eta$-dependent ${ }^{1} E_{\mathrm{T}}$ threshold and hadronic isolation requirements. For Run 2, some improvements that have been made for selecting EM objects at L1 include the use of L1 EM isolation, upgrades to the L1 calorimeter hardware for applying dynamic pileup corrections, and an increase to the $\mathrm{L} 1 \mathrm{maximum}$ readout rate from $75 \mathrm{kHz}$ to $100 \mathrm{kHz}$. There are also new $\mathrm{L} 1$ topological triggers being commissioned that will help to reduce rates by selecting specific event topologies based on angular separation and invariant mass requirements. In particular, these topological triggers can be used for $J / \psi \rightarrow e e$ and $W \rightarrow e v$ tag-and-probe ${ }^{2}$ triggers, which would otherwise have larger rates at $\mathrm{L} 1$.

Events which pass the L1 trigger selection are then processed by the software-based high level trigger (HLT). In Run 1, the HLT was composed of two steps known as Level 2 (L2) and the Event Filter; these have been merged into a single HLT step for Run 2. This allows for more flexibility, the use of more complex algorithms, and better harmonization between the objects reconstructed in the trigger (online) and those ultimately reconstructed with the final processing (offline). Some additional improvements specific to electrons and photons that have been made at the HLT for Run 2 include improvements to the HLT cluster and track reconsruction algorithms, the use of a new multivariate algorithm for energy calibration to improve the EM energy measurement in the trigger, and reoptimization of the electron and photon identification (ID) algorithms at the HLT.

\section{Electron and Photon Identification}

Electron and photon candidates are reconstructed in the ATLAS detector as clusters of energy deposited in the EM calorimeter, where electrons additionally have an associated track in the inner detector. To determine whether these electron and photon candidates are signal-like objects or background-like objects such as hadronic jets, efficient algorithms for electron and photon ID are

\footnotetext{
${ }^{1}$ ATLAS uses a right-handed coordinate system with its origin at the nominal $p p$ interaction point at the center of the detector. The positive $x$-axis is defined by the direction from the interaction point to the center of the LHC ring, with the positive $y$-axis pointing upwards, while the beam direction defines the $z$-axis. The azimuthal angle $\phi$ is measured around the beam axis and the polar angle $\theta$ is the angle from the $z$-axis. The pseudorapidity is defined as $\eta=-\ln \tan (\theta / 2)$. Transverse energy is computed as $E_{\mathrm{T}}=E \cdot \sin (\theta)$.

${ }^{2}$ The tag-and-probe method relies on the the decay products of resonances such as the $W, Z$, or $J / \psi$, and is used to select unbiased electron candidates (probes) by applying strict requirements on the other object in the decay (tags).
} 
applied. Electron and photon ID make extensive use of calorimeter shower shapes and widths, energy ratios, information from the transition radiation tracker, track-cluster matching, and tracking quantities [3]. Distributions of these discriminating quantities, measured using data collected in June and July 2015, can be found in [4].

The electron ID in the ATLAS trigger is performed using both likelihood (LH) and selectioncriteria-based variants, while the photon ID is exclusively selection-criteria-based. In a selectioncriteria-based ID, fixed requirements are imposed on quantities that discriminate between signal and background. The LH ID, by contrast, is based on probability distribution functions (PDFs) of these various quantities for electrons and background processes. These PDFs are then used to determine the probability for a given electron candidate to be signal or background, and these probabilities are combined to form a likelihood discriminant. Finally, a requirement is imposed on the likelihood discriminant to select signal-like objects and reject background-like objects [3]. Typically three identification operating points are used for both electron and photon ID. These operating points are referred to, in order of increasing background rejection, as Loose, Medium, and Tight.

In Run 1, the electron trigger only used a selection-criteria-based ID, while LH and selectioncriteria-based electron ID both existed offline. These were both designed to have the same signal efficiency for a given operating point, which resulted in a background rejection that was approximately a factor of two better for the LH than the selection-criteria-based ID, as seen in Figure 1. As a result, the LH has become the primary method of electron ID for ATLAS in Run 2. Furthermore, as inefficiencies can arise by applying a LH selection offline with a selection-criteria-based selection in the trigger, the LH electron ID was adapted for the trigger and is now the default. A selection-criteria-based trigger exists as backup during commissioning, but will not be supported beyond that.
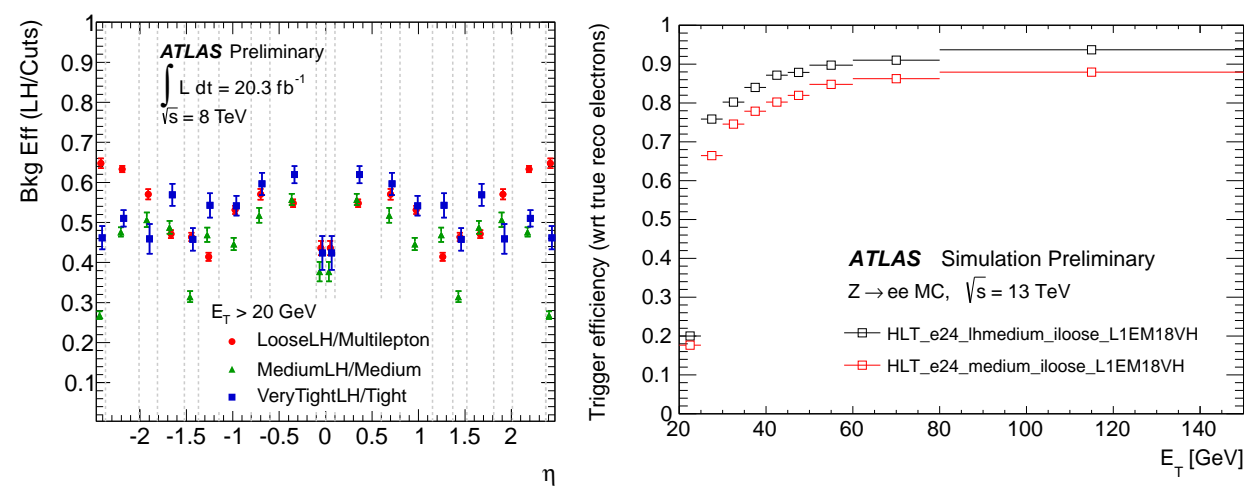

Figure 1: Left: Ratio of background efficiencies in Run 1 for LH operating points with respect to corresponding selection-criteria-based operating points, where each ratio compares operating points which have approximately the same signal efficiency. Thus, for a given signal efficiency, the LH operating points reduce backgrounds by about a factor of two with respect to the selection-criteria-based operating points, on average [3]. Right: The Run 2 absolute trigger efficiencies expected for the HLT_e24_(lh)medium_iloose_L1EM18VH triggers as a function of the $E_{\mathrm{T}}$ of the offline electron candidate, measured with respect to reconstructed electrons originating from the $Z \rightarrow e e$ decay in simulation. This trigger requires an electron candidate to have $E_{\mathrm{T}}>24 \mathrm{GeV}$, to satisfy the appropriate selection-criteria-based or LH identification criteria for the Medium operating point, and to pass a loose isolation requirement [5]. 


\section{Results and Trigger Performance}

\subsection{Electron Efficiencies and Rates}

While the Run $1 \mathrm{LH}$ operating points were each designed to have the same absolute signal efficiency as each corresponding selection-criteria-based operating point, this strategy was not chosen for the Run 2 operating points due to rate requirements. Instead, the LH signal efficiency is about $6 \%$ higher than that of the selection-criteria-based triggers, shown in Figure 1. As seen in Figure 2, the trigger efficiencies measured with respect to the corresponding offline ID for the $\mathrm{LH}$ and selection-criteria-based triggers are similar. Additionally, the rates for the LH triggers are about $20 \%$ lower than those of the selection-criteria-based triggers, while the Tight electron triggers have rates that are about 50\% lower than those for the Medium triggers.
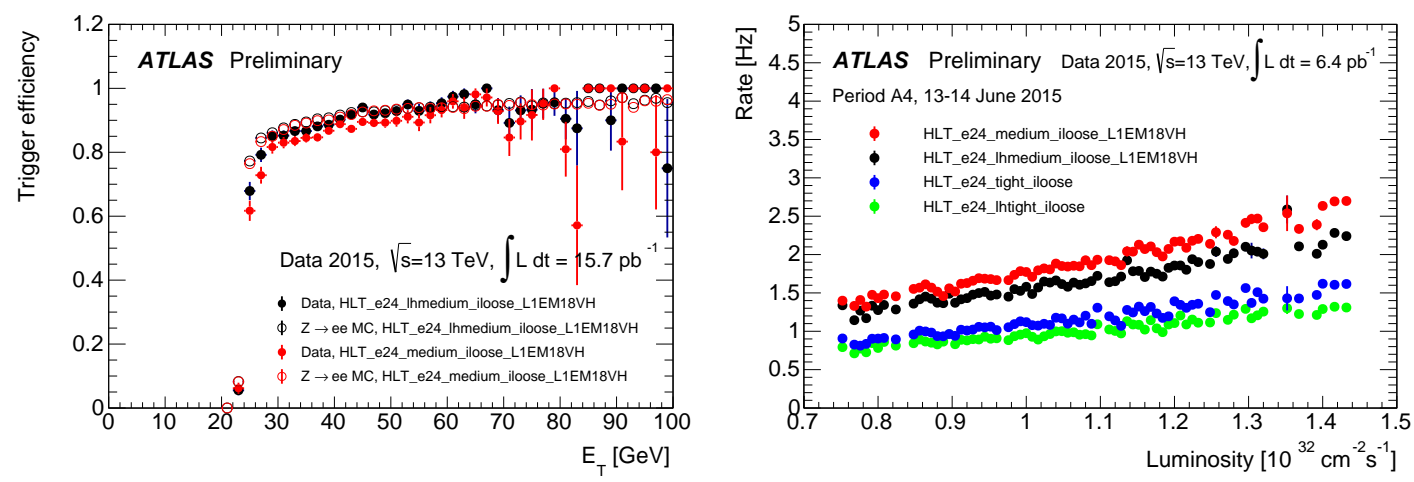

Figure 2: Left: The single-electron trigger efficiencies for the HLT_e24_(lh)medium_iloose_L1EM18VH triggers as a function of the offline electron candidate's $E_{\mathrm{T}}$, measured with respect to the corresponding offline electron ID using data collected in June and July 2015. Right: Output rates for the HLT_e24_(lh)medium_iloose_L1EM18VH and HLT_e24_(lh)tight_iloose triggers as a function of the instantaneous luminosity, measured with data collected in June 2015 [5].

\subsection{Photon Efficiencies and Rates}

Unlike the primary single-electron triggers discussed in Section 3.1, the single-photon triggers that will now be discussed are referred to as supporting (or prescaled) triggers, which are only used to collect some fraction of the events which pass all of the requirements for the given trigger. This is done to reduce rates for signatures where it is either not necessary or not feasible to record every event. In particular, the prescaled single-photon triggers shown in Figure 3 are useful for measuring inclusive photon production as well as for studying the unprescaled trigger HLT_g35_medium_g25_medium, which is essential for the study of $H \rightarrow \gamma \gamma$. This diphoton trigger requires two photons to satisfy the Medium photon identification at the HLT and leading (subleading) photon $E_{\mathrm{T}}>35$ (25) GeV, so by studying precaled single-photon triggers with similar $E_{\mathrm{T}}$ thresholds and identification criteria it is possible to study the performance of the unprescaled diphoton trigger as well.

Figure 3 shows the trigger efficiencies measured with respect to the offline Tight photon ID for the Medium prescaled single-photon triggers. The rates for the Medium triggers are about $50 \%$ 
lower than for the Loose triggers, while the $35 \mathrm{GeV}$ threshold reduces the rate by a factor of 2-3 compared to the $25 \mathrm{GeV}$ threshold.
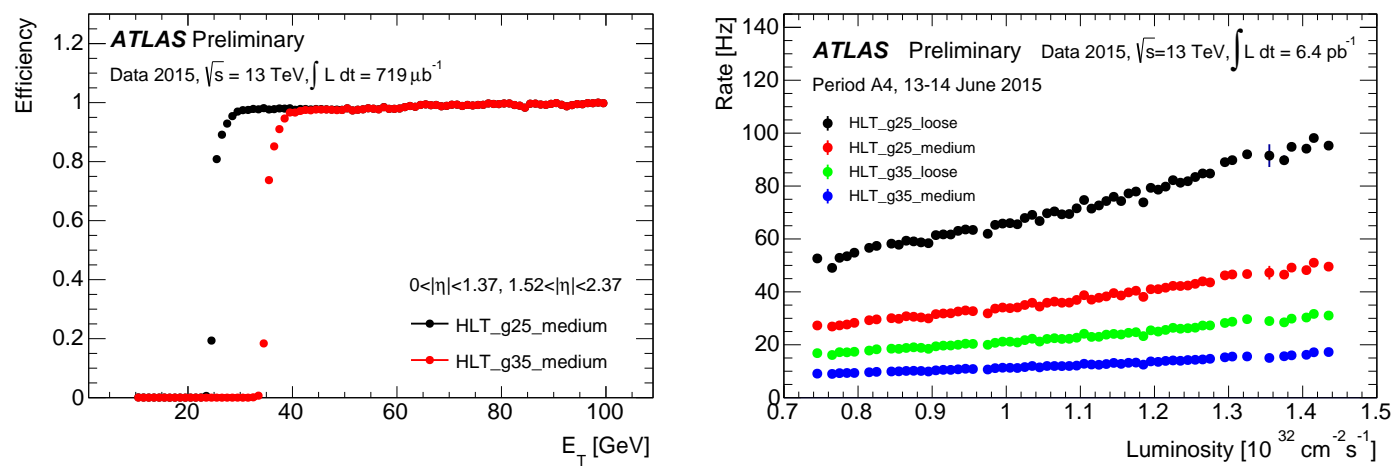

Figure 3: Left: The single-photon trigger efficiencies for the prescaled HLT_g25(35)_medium triggers, measured with respect to the Tight offline photon ID using data collected in June and July 2015. This trigger requires a photon candidate to have $E_{\mathrm{T}}>25$ (35) $\mathrm{GeV}$ and to satisfy the Medium photon ID operating point. Right: Output rates for the HLT_g25(35)_loose(medium) single-photon triggers as a function of the instantaneous luminosity, measured with data collected in June 2015 [5].

\section{Conclusions}

Many improvements have been made for the electron and photon triggers since Run 1, and improvements will continue throughout Run 2 to ensure that rates remain managable without degrading signal efficiencies. With the early Run 2 data, ATLAS is already seeing well-performing triggers as a result of the work that has been done, and physics analyses are already using the electrons and photons that have been collected thus far in Run 2.

\section{References}

[1] ATLAS Collaboration, The ATLAS Experiment at the CERN Large Hadron Collider, JINST 3 (2008) S08003.

[2] ATLAS Collaboration, Technical Design Report for the Phase-I Upgrade of the ATLAS TDAQ System, Tech. Rep. CERN-LHCC-2013-018. ATLAS-TDR-023, CERN, Geneva, 2013.

https://cds.cern. ch/record/1602235.

[3] ATLAS Collaboration, Electron efficiency measurements with the ATLAS detector using the 2012 LHC proton-proton collision data, ATLAS-CONF-2014-032, 2014.

http: / / cdsweb. cern. ch/record/1706245.

[4] ATLAS Collaboration, Electron shower shapes, tracking, isolation and invariant mass distributions from $Z \rightarrow e e$ and $\mathrm{J} / \psi \rightarrow$ ee events, https://atlas.web. cern. ch/Atlas/GROUPS/PHYSICS/PLOTS/EGAM-2015-003.

[5] ATLAS Collaboration, Public Egamma Trigger Plots for Collision Data, https://twiki.cern. ch/twiki/bin/view/AtlasPublic/EgammaTriggerPublicResults. 